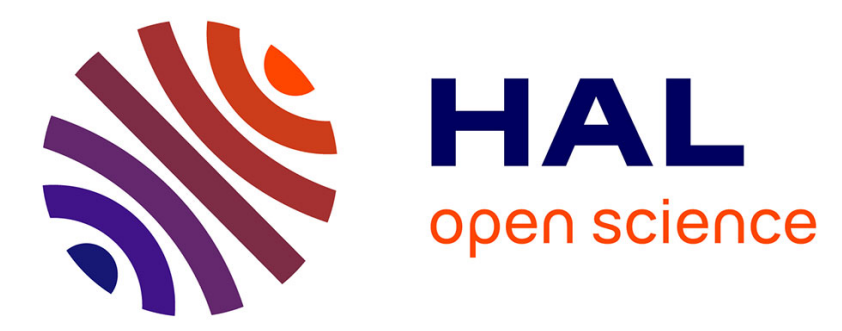

\title{
Optimisation des conditions d'élaboration du silicium photovoltaïque par fusion de zone sous plasma
}

J. Amouroux, D. Morvan, C. Sauvestre, G. Revel, M. Fedoroff, J.C. Rouchaud

\section{To cite this version:}

J. Amouroux, D. Morvan, C. Sauvestre, G. Revel, M. Fedoroff, et al.. Optimisation des conditions d'élaboration du silicium photovoltaïque par fusion de zone sous plasma. Revue de Physique Appliquée, 1980, 15 (7), pp.1239-1249. 10.1051/rphysap:019800015070123900 . jpa-00244846

\section{HAL Id: jpa-00244846 https://hal.science/jpa-00244846}

Submitted on 1 Jan 1980

HAL is a multi-disciplinary open access archive for the deposit and dissemination of scientific research documents, whether they are published or not. The documents may come from teaching and research institutions in France or abroad, or from public or private research centers.
L'archive ouverte pluridisciplinaire HAL, est destinée au dépôt et à la diffusion de documents scientifiques de niveau recherche, publiés ou non, émanant des établissements d'enseignement et de recherche français ou étrangers, des laboratoires publics ou privés. 


\title{
Optimisation des conditions d'élaboration du silicium photovoltaïque par fusion de zone sous plasma
}

\author{
J. Amouroux, D. Morvan, C. Sauvestre \\ Laboratoire de Génie Chimique, E.N.S.C.P., 11 rue Pierre-et-Marie-Curie, 75231 Paris Cedex 05, France
}

G. Revel, M. Fedoroff et J. C. Rouchaud

Laboratoire de Radioactivation, Centre d'Etude de Chimie Métallurgique, 15 rue Georges-Urbain, 94400 Vitry/Seine, France

(Reçu le 29 janvier 1980, révisé le 17 mars 1980, accepté le 1 er avril 1980)

\begin{abstract}
Résumé. - La technique de fusion de zone sous plasma est un procédé de purification qui permet d'obtenir un silicium photovoltaïque à partir d'un silicium métallurgique. La méthode consiste à placer un barreau de silicium sous un plasma d'argon-hydrogène, de provoquer ainsi la fusion d'une zone que l'on déplace afin de drainer les impuretés en surface et en queue de barreau. Un décapage de la surface à l'acide $\left(\mathrm{HF}-\mathrm{HNO}_{3}\right)$ permet d'éliminer les impuretés entre chaque passage. En fin de traitement la concentration en impureté est inférieure à 1 ppm. Nous précisons que le transfert de masse est indépendant de la vitesse de déplacement de la zone liquide, qui est voisine de $40 \mathrm{~cm} / \mathrm{h}$, mais dépend de la vitesse de transfert de chaleur entre le plasma et le silicium solide. Du point de vue fondamental elle exige de revoir les modèles de purification par fusion de zone classique qui ne s'appliquent plus à la technique plasma.
\end{abstract}

\begin{abstract}
Plasma melting zone technique is a process that permits the direct purification of metallurgical-grade silicon to photovoltaic-grade silicon. This technique consists of placing a bar of silicon under the influence of argon-hydrogen plasma which produces a melted zone, and displacing it along the bar to drain impurities on to the surface and to the end of the bar. An acidic treatment $\left(\mathrm{HF}-\mathrm{HNO}_{3}\right)$ is applied then to eliminate the impurities between each passage of the zone. At the end of the operation a concentration of impurities less than $1 \mathrm{ppm}$ is obtained. It has been proved that mass transfer is independent of the rate of displacement wich is around $40 \mathrm{~cm} / \mathrm{h}$, but dependent on the rate of heat transfer. From a fundamental point of view, this technique necessitates the reconsideration of classical mathematic models wich are no longer valied for plasma melting zone processes.
\end{abstract}

1. Introduction. - La technique de fusion de zone sous plasma apparaît capable d'élaborer du silicium très pur à partir du silicium métallurgique. Notre but est d'atteindre une pureté photovoltaïque dans des conditions techniques compatibles avec une application industrielle. Il s'agit donc d'étudier de façon systématique les variables d'action qui contrôlent le fonctionnement du procédé. Rappelons que nous avons montré le rôle bien particulier des deux interfaces (liquide-solide, et liquide-gaz) et des phénomènes de transfert au travers de la goutte liquide : transfert de chaleur et transfert de matière.

Le phénomène d'extraction des impuretés de l'état solide consiste à les drainer au travers de la goutte liquide vers la surface du matériau. Dans ces conditions il devient indispensable d'analyser les phénomènes physicochimiques qui contrôlent le transfert de matière [1-14]. Ils dépendent en premier du silicium liquide : viscosité et coefficient de diffusion, mais également du gradient thermique qui caractérise le transfert d'énergie entre le plasma et le silicium liquide. En second il dépendent des caractéristiques de l'interface d'extraction liquide-solide c'est-à-dire des coefficients de partage, de l'épaisseur de la couche de diffusion [15-27]. Enfin la géométrie de la goutte liquide en phase plasma provoque d'une part une augmentation de la surface de l'interface solideliquide et d'autre part une agitation importante par des processus convectifs.

Ainsi les transferts de matière sont-ils contrôlés par des mécanismes convectifs dépendant des gradients de températures et de concentrations d'une part et de la viscosité du liquide d'autre part.

La présentation de cette deuxième partie comportera 3 étapes, tout d'abord le rôle des variables d'action 
qui contrôlent le processus expérimental de purification sous plasma, en second lieu, l'analyse des grandeurs physicochimiques responsables des mécanismes de transfert de matière et de chaleur à l'intérieur de la goutte, enfin une présentation du silicium photovoltaïque élaboré et les conditions optimales de traitement réalisé. Le plan de notre étude peut se résumer à partir du tableau $I$ et du modèle macroscopique de purification (Fig. 1).

2. Etude expérimentale des paramètres de fonctionnement de la zone fondue sous plasma. - A partir des premières constatations, l'étude expérimentale de la zone fondue sous plasma a été réalisée afin de définir le rôle de chacune des caractéristiques de la méthode :

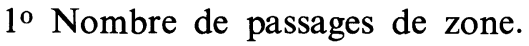

$2^{\circ}$ Vitesse de déplacement.

30 Traitement acide.

$4^{\circ}$ Nature du matériau de départ.

$5^{\circ}$ Renouvellement du métal de queue.

$6^{\circ}$ Longueur du barreau et la largeur de zone.

L'analyse de ces résultats soulignera l'amélioration des processus d'échange apportée par le chauffage au plasma en fusion de zone.

Nous conclurons par l'interprétation du processus de purification en tenant compte de l'action conjuguée de l'ensemble des phénomènes ayant lieu dans la zone liquide.

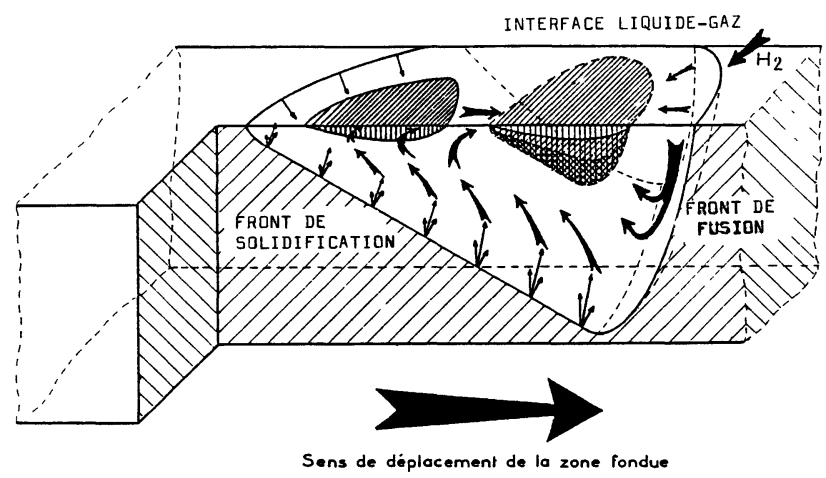

Fig. 1. - Rappel de la géométrie de la zone liquide en fusion de zone sous plasma.

[Geometry of liquid zone in plasma melting zone process.]

2.1 INFLUENCE DU NOMBRE DE PASSAGES DE ZONE. Les constatations expérimentales concernant la section du barreau fondu nécessitent d'effectuer un nombre pair de passage soit 2, 4, 6 ou 8 .

Les mesures (Figs. 2 et 3 ) du gradient de concentration des impuretés le long du barreau indiquent que deux passages permettent une diminution de la concentration des impuretés en tête de barreau d'un facteur 100 à 1000 . Cette purification se prolonge sur la moitié de la longueur de barreau de façon uniforme au bout de 6 passages. L'augmentation du nombre de passages déplace la position de ce palier de concentration vers des teneurs plus faibles, et augmente sa longueur.

Tableau I. - Analyse paramétrique de la purification du silicium par fusion de zone sous plasma.

[Parametric analysis of the purification of silicon by plasma melting zone.]

Variables d'action

Grandeurs physicochimiques

A l'interface solide-liquide :

Surface d'échange

Coefficient de diffusion

- Vitesse de déplacement de la zone liquide

- Influence du plasma

- Augmentation du nombre de passages

- Décapage de la surface entre chaque passage
Epaisseur de la couche de diffusion

Coefficient de partage effectif $k$

Gradient thermique

Gradient de $\{$ vertical

concentration $\{$ horizontal

Dans le liquide :

Température du liquide

Coefficient de diffusion

Viscosité du liquide

Gradient thermique

Gradient de concentration

A l'interface gaz-liquide :

Elimination des impuretés par augmen-

tation des tensions de vapeur

Surface d'échange

Elimination des impuretés par réaction chimique
Caractéristiques du matériau

- Pureté

- Aspect cristallin par micrographie

- Mesures physiques 

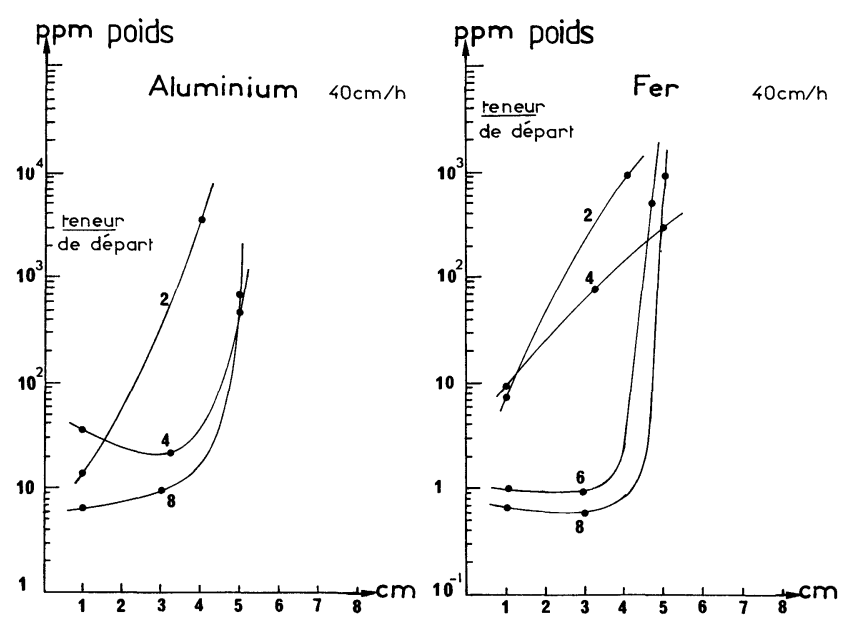

Figs. 2 et 3 . - Influence du nombre de passages sur la purification obtenue sans décapage de la surface par l'acide.

[Influence of the number of pass on the purification obtained without acid treatement.]

D'après la théorie, le premier passage en fusion de zone classique est le plus efficace ; ensuite la pureté s'améliore de plus en plus lentement jusqu'à ce qu'une répartition limite soit atteinte. Dans le cas de la fusion de zone. sous plasma, l'état d'équilibre n'est jamais atteint car les impuretés sont drainées en surface et peuvent subir une extraction par l'atmosphère chaude et réactive du plasma. Ce fait explique l'amélioration importante apportée par l'augmentation du nombre de passages. Toutefois les mesures effectuées en tête de barreau indiquent une concen- tration en impuretés supérieure à celle du milieu de barreau. Ce fait qui pourrait être expliqué par le drainage d'impuretés inverses provient en fait de la perturbation du front de solidification au départ de la fusion de zone. La goutte de départ présente une fusion totale jusqu'au fond de la nacelle avec drainage des impuretés sur la zone inférieure du barreau. Cette perturbation du front de solidification disparaît après $1 \mathrm{~cm}$ de traitement.

2.2 INFLUENCE DE LA VITESSE DE DÉPLACEMENT. Le principe expérimental de la fusion de zone précise que la vitesse de déplacement de la zone liquide doit être suffisamment lente pour permettre la diffusion complète des impuretés dans la zone liquide; il est nécessaire de surcroît d'apporter la plus grande attention à la régularité du déplacement du front de solidification, surtout aux faibles vitesses afin d'éviter la perturbation de la couche de solidification. Nos essais en fusion de zone sous plasma ont été effectués à des vitesses de 20,30 et $40 \mathrm{~cm} / \mathrm{h}$.

L'analyse du gradient de concentration des impuretés le long du barreau après 2 à 4 passages montre (Figs. 4 et 5) que la vitesse n'est pas un paramètre qui produit une grande modification du drainage des impuretés vers la queue du barreau. Il faut donc admettre que la modification des paramètres physicochimiques liés aux très hautes températures de surface accélère la cinétique de drainage des impuretés puisque nos essais sont effectués à des vitesses 10 à 100 fois supérieures à celles usuellement employées. Nous essaierons de mesurer ultérieurement l'évolution
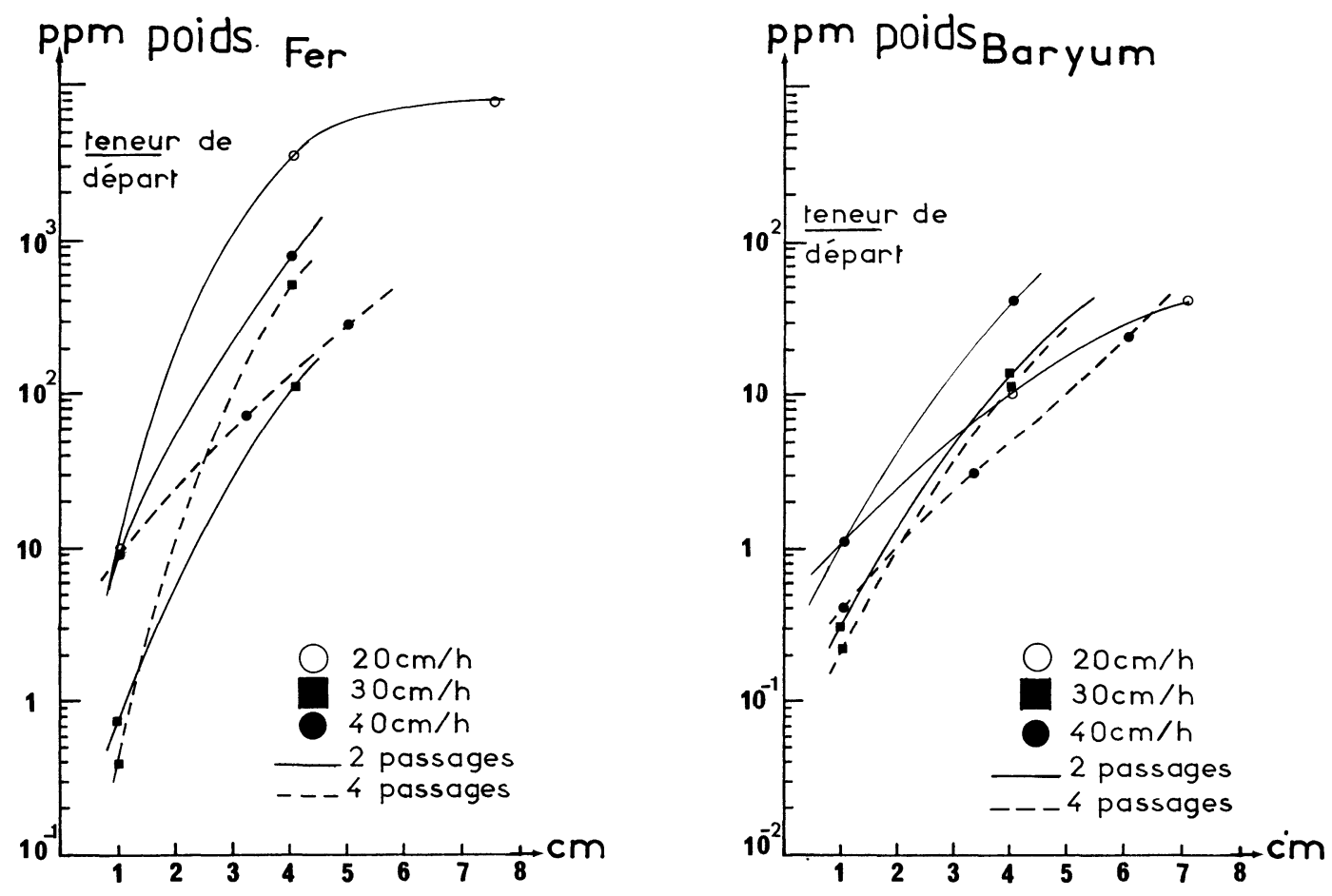

Figs. 4 et 5 . Influence de la vitesse sur la purification obtenue sans décapage de la surface par l'acide.

[Influence of the rate of zone travel on the purification obtained without acid treatement.] 
des grandeurs physicochimiques (épaisseur du film diffusionnel interfacial, viscosité, coefficient de diffusion et de partage) afin de conclure plus nettement sur le rôle de la vitesse.

Nous avons tenté d'employer des vitesses supérieures à $40 \mathrm{~cm} / \mathrm{h}$; toutefois, dans nos conditions d'expériences, le transfert thermique n'avait "pas le temps de s'établir de sorte que la fusion n'était pas homogène. Toutefois ce paramètre devrait faire l'objet d'une optimisation plus poussée dans le cadre d'une expérimentation sur des, barreaux de section plus importante.

2.3 Influence du TRAitement aCide. - Comme nous l'avons montré la fusion de zone au plasma permet outre le drainage de toutes les impuretés en queue de barreau, un drainage préférentiel vers la surface. Aussi un traitement dans le bain acide $\left(\mathrm{HNO}_{3} 50 \%, 4 / 5\right.$ en volume et $\mathrm{HF} 50 \%, 1 / 5$ en volume) permet-il d'éliminer de la masse une quantité d'impureté très importante.

Cette attaque déplace le gradient limite de concentration vers des valeurs faibles (Figs. 6 et 7). Elle se traduit par une amélioration très nette de la pureté obtenue et une augmentation de la longueur purifiée. Ainsi un barreau ayant subi 4 passages avec traitement acide intermédiaire a un profil de concentration équivalent à un échantillon 8 passages non traité. Cette amélioration est particulièrement nette pour les impuretés dont la concentration de départ est élevée. Ce résultat confirme le mécanisme de drainage des impuretés vers la surface du barreau et indique l'importance du mécanisme d'extraction vers l'interface liquide-gaz.

2.4 INFLUENCE DU MATÉRIAU DE DÉPART. - Le silicium métallurgique contient des impuretés bien différentes selon lesable de départ choisi, le réducteur employé (coke ou charbon de bois) et le niveau de la

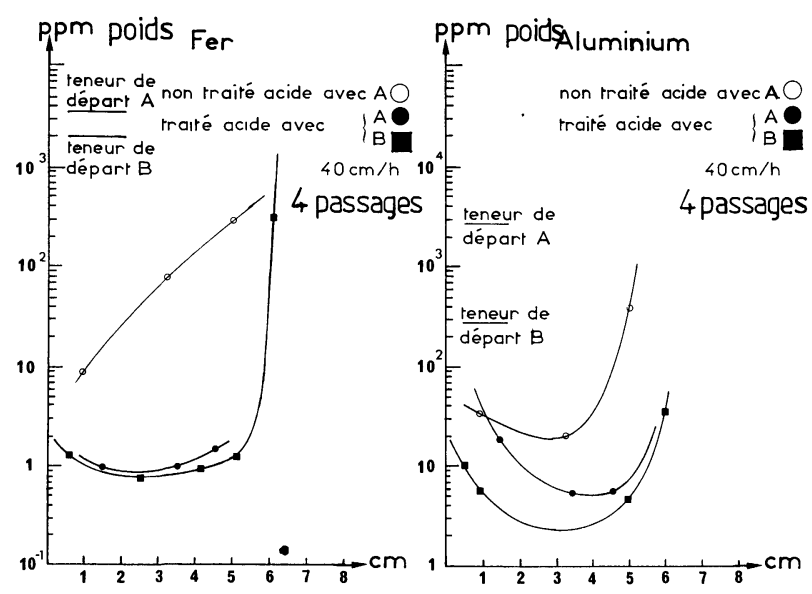

Figs. 6 et 7 . Influence du décapage de la surface par l'acide et du choix du matérau de départ sur la purification obtenue avec 4 passages à $40 \mathrm{~cm} / \mathrm{h}$.

[Influence of acid treatement and the choice of metallurgical silicon on the purification obtained with 4 pass at $40 \mathrm{~cm} / \mathrm{h}$.] coulée retenu après traitement en four électrique. C'est ainsi que l'emploi de siliciums métallurgiques d'origine et de pureté différentes permet une amélioration de la purification pour le matériau de départ ayant la plus faible teneur en impureté (Tableau II) (Figs. 6-7). Toutefois dans le cas de l'aluminium, l'amélioration n'est pas aussi nette que prévue (Fig. 7). En effet il faut rappeler que l'analyse de l'aluminium dans le silicium par radioactivation est arrivée à sa limite de détection à partir de 5 ppm poids ; au voisinage de cette concentration il est admis que celle-ci est inférieure à la ppm. En effet la réaction nucléaire $(28 \mathrm{Si} \mathrm{n}, \mathrm{p})(28 \mathrm{Al})$ conduit au même radio-isotope que celui utilisé pour le dosage de l'aluminium soit $(27 \mathrm{Al} \mathrm{n}, \gamma)(28 \mathrm{Al})$.

Tableau II. - Analyse des matériaux de départ par : $\left.\begin{array}{l}\text { Radioactivation } \\ \text { * Spectrométrie de masse }\end{array}\right\}$ en ppm poids.

[Elements analysis of the metallurgical silicon by : $\left.\begin{array}{l}\text { Radioactivation } \\ \text { *Mass spectrometry }\end{array}\right\}$ in ppm weight.]

\begin{tabular}{ccc} 
Eléments & $\begin{array}{c}\text { Silicium } \\
\text { métallurgique A }\end{array}$ & $\begin{array}{c}\text { Silicium } \\
\text { métallurgique }\end{array}$ \\
\hline $\mathrm{Al}$ & - & - \\
$\mathrm{Ag}$ & 2800 & 290 \\
$\mathrm{As}$ & 0,7 & $<0,06$ \\
$\mathrm{Ba}$ & 0,21 & 0,03 \\
$\mathrm{Co}$ & 20 & 10 \\
$\mathrm{Cr}$ & $120^{*}$ & 3,5 \\
$\mathrm{Cl}$ & 9 & 4,3 \\
$\mathrm{Cu}$ & 74 & $<73$ \\
$\mathrm{Fe}$ & $75^{*}$ & 14 \\
$\mathrm{~K}$ & $240^{*}$ & 2000 \\
$\mathrm{Mn}$ & $40^{*}$ & 3 \\
$\mathrm{Mg}$ & 579 & 160 \\
$\mathrm{Mo}$ & $180^{*}$ & $<100$ \\
$\mathrm{Na}$ & 84 & $<2$ \\
$\mathrm{Sb}$ & $140^{*}$ & 0,1 \\
$\mathrm{~V}$ & $460^{*}$ & 0,02 \\
$\mathrm{~W}$ & $10^{*}$ & 13,3 \\
& $6,4^{*}$ & 0,2
\end{tabular}

2.5 INFLUENCE DU RENOUVELLEMENT DU MÉTAL DE QUEUE APRÈS UN CERTAIN NOMBRE DE PASSAGES. - Afin d'éviter la saturation en impuretés en queue de barreau il est possible de remplacer celle-ci après un certain nombre de passages par un barreau purifié. Il faut noter que le silicium a l'avantage de n'avoir que des impuretés directes ainsi la question du remplacement du métal de queue dépourvu d'impuretés inverses ne se pose pas. L'expérience a été réalisée avec deux barreaux traités, 4 passages à $40 \mathrm{~cm} / \mathrm{h}$ avec traitement acide entre chaque passage. 
Nous avons récupéré les deux moitiés de tête et effectué deux autres passages dans les mêmes conditions. Nous avons comparé la concentration en deux points de ce barreau avec un barreau ayant subi 6 passages à $40 \mathrm{~cm} / \mathrm{h}$ avec traitement acide. Nous observons non seulement une amélioration dans la première moitié du barreau mais surtout une augmentation de la longueur purifiée (Tableau III).

Tableau III. - Influence du changement de queue d̀ $40 \mathrm{~cm} / \mathrm{h}$ avec traitement acide. Résultats en ppm poids.

[Influence of tail replacement at $40 \mathrm{~cm} / \mathrm{h}$ with acid treatement.]

\begin{tabular}{|c|c|c|c|c|}
\hline \multicolumn{3}{|c|}{$d=0,5 \mathrm{~cm}$ de la tête } & \multicolumn{2}{|c|}{$d=6 \mathrm{~cm}$ de la tête } \\
\hline $\begin{array}{c}\text { Elé- } \\
\text { ments }\end{array}$ & 6 passages & $\begin{array}{c}6 \text { passages } \\
\text { avec } \\
\text { changement } \\
\text { de queue }\left(^{*}\right)\end{array}$ & 6 passages & $\begin{array}{c}6 \text { passages } \\
\text { avec } \\
\text { changement } \\
\text { de queue }\left(^{*}\right)\end{array}$ \\
\hline - & - & - & - & - \\
\hline $\mathrm{Al}$ & 4,7 & 5,4 & 870 & 7 \\
\hline V & 0,0003 & 0,008 & 20,5 & 0,005 \\
\hline $\mathrm{Mn}$ & 0,015 & 0,02 & 22,3 & 0,02 \\
\hline $\mathrm{Cl}$ & $<0,2$ & $<0,2$ & $<10$ & $<0,2$ \\
\hline $\mathrm{Mg}$ & $<2$ & $<2$ & $<12,5$ & $<2$ \\
\hline Co & 0,05 & $<0,001$ & 0,5 & 0,008 \\
\hline $\mathrm{Fe}$ & $<1$ & $<1,1$ & 177 & $<0,8$ \\
\hline $\mathrm{Ba}$ & $<0,3$ & $<0,7$ & 41 & $<0,5$ \\
\hline $\mathrm{Cr}$ & 0,03 & $<0,01$ & 2 & 0,02 \\
\hline $\mathrm{Cu}$ & $<0,03$ & $<0,03$ & 1 & $<0,05$ \\
\hline $\mathrm{Na}$ & $<0,01$ & $<0,003$ & 0,05 & $<0,003$ \\
\hline $\mathrm{Au}$ & 0,0001 & 0,0001 & 0,0006 & 0,0001 \\
\hline
\end{tabular}

$(*)$ Changement de queue après 4 passages par une tête ayant subi 4 passages. Puis traitement de 2 passages.

$\left[\left(^{*}\right)\right.$ Tail replacement after 4 pass by a head that has been passed 4 times. Then two other pass.]

2.6 INFLUENCE DE LA LONGUEUR DE BARREAU ET DE LA LARGEUR DE ZONE. - La longueur de la zone fondue définit la surface d'échange entre le plasma et la goutte liquide de sorte que son importance est fort différente de celle proposée dans la fusion de zone classique. Enfin la surface de l'interface de solidification dépend de la longueur de la zone de sorte que son augmentation améliore le processus de purification.

Les expériences réalisées avec les barreaux de $10 \mathrm{~cm}$ de long ont montré une longueur de zone fondue voisine de $1,5 \mathrm{~cm}$, dans ces conditions nous avons obtenu $7 \mathrm{~cm}$ de barreau purifié pour 4 passages à $40 \mathrm{~cm} / \mathrm{h}$ avec traitement acide. Il est probable qu'un barreau plus long permettrait d'augmenter la proportion de barreau traité et de confirmer l'intérêt de la méthode pour des applications industrielles.

2.7 CONCLUSION SUR LE MODÈLE DE FUSION DE ZONE SOUS PLASMA. - L'examen des variables d'action qui permettent le contrôle du fonctionnement de la zone fondue montre que leur poids respectif est très différent du modèle classique, corroborant ainsi l'analyse du phénomène tel qu'il était déjà apparu dans la première étude ; les paramètres clés sont le traitement acide et le nombre de passages. Par contre les paramètres vitesse de déplacement et largeur de zone n'ont qu'un rôle mineur et difficile à mesurer dans nos expériences.

Ces faits confirment le rôle déterminant des processus convectifs qui présentent la fusion de zone sous plasma comme un mécanisme de drainage des impuretés de l'interface liquide-solide vers l'interface liquide-gaz : il s'agit donc d'un mécanisme d'extraction liquide-solide. Les mécanismes diffusionnels jouent en fait un rôle secondaire et la vitesse de traitement peut donc être considérablement augmentée. Pour confirmer ces conclusions nous allons entre autres calculer les gradients de concentration à l'interface solide-liquide et comparer l'évolution d'un profil de concentration théorique et expérimental d'une impureté le long du barreau. Ces calculs nous permettent d'établir des corrélations entre valeurs théoriques et résultats expérimentaux afin de définir les conditions optimales de traitement.

3. Calculs théoriques des grandeurs physicochimiques dans la fusion de zone sous plasma. Corrélation entre les valeurs théoriques et les résultats expérimentaux. L'analyse des grandeurs physicochimiques associées au processus de purification peut être réalisée en 3 étapes.

1) Caractéristiques des milieux liquides : viscosité, densité, coefficient de diffusion.

2) Caractéristiques de l'interface solide-liquide : coefficient de partage, épaisseur de la couche de diffusion, influence de la géométrie de la goutte liquide sur le gradient de concentration.

3) Comparaison entre profil de concentration théorique et expérimental.

Toutes ces grandeurs ont en commun leur très forte dépendance de la température, paramètre clé $\mathrm{du}$ processus de purification [19-20].

3.1 ANALYSE DES CARACTÉRISTIQUeS DU SILICIUM LIQUIDE. - 3.1.1 Viscosité. - La connaissance de la viscosité [28-31] du silicium fondu est nécessaire pour la détermination du coefficient de diffusion et la mesure des mouvements convectifs existant dans la zone. La variation de la viscosité du silicium liquide en fonction de la température n'est connue que de $1420^{\circ} \mathrm{C}$ à $1660^{\circ} \mathrm{C}$. A partir de l'hypothèse selon laquelle la viscosité est une grandeur thermiquement activée nous avons calculé les valeurs jusqu'à $2400{ }^{\circ} \mathrm{C}$ en employant l'expression suivante

$$
\mu=A \mathrm{e}^{-H_{\eta} / R T}
$$

$\mu$ : viscosité en poise

$A$ : constante en poise

$H \eta$ : énergie d'activation de viscosité en $\mathrm{cal} / \mathrm{g}$. atome

$T$ : température en degré kelvin

$R$ : constante des gaz parfaits en cal $/ \mathrm{kg}$. atome. 
La variation de la viscosité du silicium en fonction de la température montre qu'à $1600^{\circ} \mathrm{C}$ elle est égale à celle de l'eau à $50^{\circ} \mathrm{C}$ et qu'à $2000^{\circ} \mathrm{C}$ le métal liquide a la même viscosité que l'eau à $70^{\circ} \mathrm{C}$ (Fig. 8).

3.1.2 Densité du silicium liquide. - La densité du silicium liquide influe sur le transfert de la matière aux interfaces. Sa densité augmente de $10 \%$ au point de fusion [28]. Ainsi à proximité du point de fusionsa valeur est de $2,33 \mathrm{~g} / \mathrm{cm}^{3}$ à l'état solide et passe à $2,53 \mathrm{~g} / \mathrm{cm}^{3}$ à l'état liquide. La contraction de volume est de $10 \%$ lors du passage de l'état solide à l'état liquide.

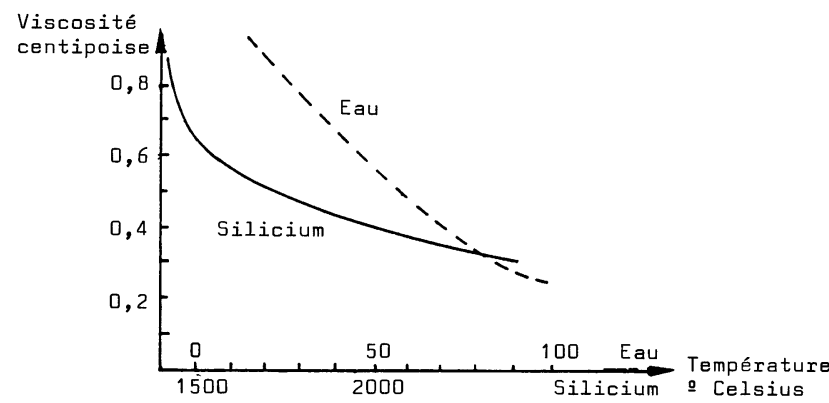

Fig. 8. - Viscosité du silicium et de l'eau en fonction de la température.

[Silicon and water viscosities as a function of temperature.]

3.1.3 Coefficients de diffusion des impuretés dans le silicium liquide. - Le coefficient de diffusion d'une impureté dans le silicium liquide permet de prévoir les transferts de matière dans cette phase [32]. Il intervient dans l'expression du calcul du coefficient de partage effectif qui régit le drainage de l'impureté dans le silicium liquide; au-delà du point de fusion, ce coefficient a été calculé à l'aide de l'expression :

$$
D_{\mathrm{AB}}=\frac{7,4 \times 10^{-8}(M)^{0,5} T}{\mu V_{\mathrm{L}}^{0,6}} \quad[33] \text {. }
$$

$D_{\mathrm{AB}}$ : coefficient de diffusion de l'impureté A dans le silicium (B) en $\mathrm{cm}^{2} / \mathrm{s}$

$M$ : masse molaire du silicium liquide $\mathrm{g} / \mathrm{mole}$

$T$ : température en degré Kelvin

$\mu \quad$ : viscosité du silicium liquide en centipoise

$V_{\mathrm{L}}$ : volume molaire de l'impureté à son point d'ébullition normal en $\mathrm{cm}^{3}$.

La figure 9 indique les variations des coefficients de diffusion de certaines impuretés dans le silicium liquide en fonction de la température.

3.2 ETUDE DE L'INTERFACE DE SOLIDIFICATION. 3.2.1 Calcul des coefficients de partage effectif $k$ caractérisant la purification par fusion de zone au plasma. - Les analyses ponctuelles effectuées le long des barreaux traités en zone fondue sous plasma permettent de calculer la valeur moyenne du coefficient de partage effectif. La concentration en impureté le long d'un barreau traité après un passage est donnée

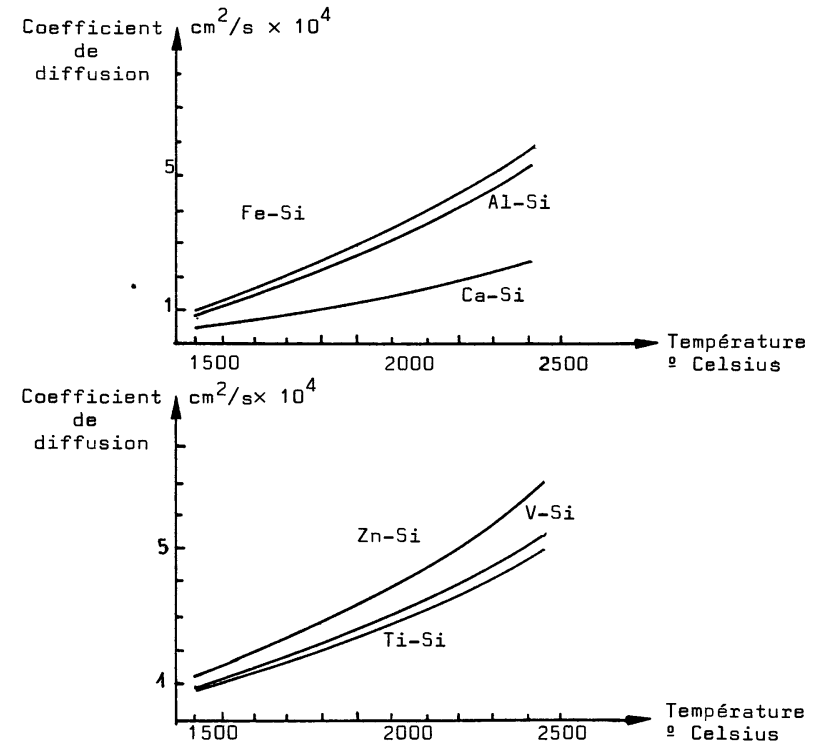

Fig. 9. - Coefficient de diffusion dans le silicium.

[Diffusion coefficient of silicon.]

par l'expression

$$
\begin{aligned}
C_{S} & =C_{0}\left[(1-k)\left(1-\mathrm{e}^{-\beta x}\right)+k\right] \\
\text { avec } \quad \beta & =\frac{k f}{D}\left[1-\exp \left(-\frac{f l}{D}\right)\right]-1
\end{aligned}
$$

$C_{0}:$ concentration initiale en $\mu \mathrm{g} / \mathrm{g}$

$C_{S}:$ concentration en fonction de $x$ en $\mu \mathrm{g} / \mathrm{g}$

$x$ : distance à partir de la tête du barreau en $\mathrm{cm}$

$k$ : coefficient de partage effectif

$D$ : coefficient de diffusion au point de fusion dans le liquide en $\mathrm{cm}^{2} / \mathrm{s}$

$f$ : vitesse de déplacement de la zone liquide en $\mathrm{cm} / \mathrm{s}$ $l$ : largeur de zone, prise égale à $1 \mathrm{~cm}$.

L'expression mathématique indiquée précédemment est utilisée en zone fondue classique pour caractériser la répartition théorique des impuretés le long du barreau après le premier passage. En zone fondue sous plasma l'expérience a montré que la totalité de la section n'est fondue qu'après le second passage. Nous avons néanmoins utilisé cette équation pour calculer le coefficient de partage effectif en prenant les concentrations ponctuelles obtenues lorsque toute la section était traitée c'est-à-dire deux passages.

Les valeurs calculées des coefficients effectifs sont très proches des coefficients de partage théorique à l'équilibre $K_{0}$ (Tableau IV).

Pour l'arsenic et l'antimoine le coefficient de partage effectif est meilleur que le coefficient de partage théorique. Ces éléments étant très volatils leur élimination par le plasma est très importante et explique l'écart entre l'expérience et le calcul. Ainsi malgré les vitesses de passage très rapides, le coefficient de partage à l'interface est favorable au processus de fusion de zone sous plasma. 
Tableau IV. - Valeurs calculées du coefficient de partage effectif et de l'épaisseur de la couche de diffusion dans les conditions suivantes : $F=11 \times 10^{-3} \mathrm{~cm} / \mathrm{s} ; P=8 \mathrm{~kW} ;$ Débit $\mathrm{Ar}=301 / \mathrm{min}$.

[Values of effective distribution coefficient and thickness of diffusion layer calculated under the following conditions : $F=11 \times 10^{-3} \mathrm{~cm} / \mathrm{s} ; P=88 \mathrm{~kW}$; Argon flow rate $=301 / \mathrm{min}$.]

\begin{tabular}{|c|c|c|c|c|}
\hline Eléments & $\begin{array}{c}\text { Coefficient } \\
\text { de diffusion } \\
\text { au point de fusion } \\
D\left(10^{4} \mathrm{~cm}^{2} / \mathrm{s}\right)\end{array}$ & $\begin{array}{c}\text { Coefficient } \\
\text { de partage } \\
\text { à l'équilibre } \\
K_{0}\end{array}$ & $\begin{array}{l}\text { Coefficients } \\
\text { de partage effectif } \\
\text { calculés }\end{array}$ & $\begin{array}{c}\text { Epaisseur } \\
\text { de la couche } \\
\text { de diffusion } \\
\delta_{\mathrm{s}}(\mathrm{cm})\end{array}$ \\
\hline - & - & - & - & - \\
\hline $\mathrm{Al}$ & 0,97 & $2 \times 10^{-3}$ & $3,9 \times 10^{-3}$ & $6 \times 10^{-3}$ \\
\hline As & 0,98 & 0,3 & $3,9 \times 10^{-4}$ & \\
\hline Co & 1,04 & $8 \times 10^{-6}$ & $4,1 \times 10^{-4}$ & $7,4 \times 10^{-2}$ \\
\hline $\mathrm{Cr}$ & 1,04 & $10^{-5}$ & $2,4 \times 10^{-4}$ & $3,0 \times 10^{-2}$ \\
\hline $\mathrm{Fe}$ & 1,05 & $8 \times 10^{-6}$ & $2,7 \times 10^{-4}$ & $3,4 \times 10^{-2}$ \\
\hline $\mathrm{Mn}$ & 1,04 & $10^{-5}$ & $6,9 \times 10^{-4}$ & $4,0 \times 10^{-2}$ \\
\hline $\mathrm{Sb}$ & 0,91 & $2,3 \times 10^{-2}$ & $1,7 \times 10^{-4}$ & \\
\hline $\mathrm{Zn}$ & 1,24 & $10^{-5}$ & $3,6 \times 10^{-5}$ & $1,4 \times 10^{-2}$ \\
\hline
\end{tabular}

3.2.2 Epaisseur de la couche de diffusion. - A l'interface de solidification, l'efficacité des échanges est étroitement liée à l'épaisseur de la couche de diffusion. Compte tenu des différentes valeurs calculées précédemment (Tableau IV) nous avons pu accéder à l'épaisseur de la couche de diffusion avec l'expression :

$$
K=\frac{k_{0}}{k_{0}+\left(1-k_{0}\right) \exp \left(-\frac{\delta f}{D}\right)}
$$

$k$ : coefficient de partage effectif

$k_{0}$ : coefficient de partage à l'équilibre

$f$ : vitesse de déplacement de la zone liquide $\mathrm{cm} / \mathrm{s}$

$\delta$ : épaisseur de la couche de diffusion en $\mathrm{cm}$

$D$ : coefficient de diffusion $\mathrm{cm}^{2} / \mathrm{s}$.

Les épaisseurs de couches de diffusion calculées à partir des résultats expérimentaux sont inférieures aux épaisseurs trouvées en fusion de zone classique (environ $10^{-1} \mathrm{~cm}$ ). Dans le cas de la fusion de zone sous plasma la présence d'un gradient thermique élevé à l'interface et de mouvements convectifs importants permet de diminuer l'épaisseur de la couche de diffusion et d'améliorer ainsi les échanges à l'interface solide-liquide.

3.2.3 Influence de la géométrie de la goutte. Calcul du gradient de concentration à l'interface solide-liquide. - La théorie de la zone fondue classique indique que le gradient de concentration à l'interface de solidification a une grande influence sur le processus de purification. Nous avons tenté d'évaluer son importance en tenant compte de la géométrie de la zone liquide.

Afin de déterminer le profil de la zone liquide nous avons effectué une zone fondue à $40 \mathrm{~cm} / \mathrm{h}$ jusqu'en milieu de barreau et nous avons arrêté le chauffage tout en effectuant une trempe du liquide par l'hydrogène du plasma. L'examen macrographique de la zone permet de définir son profil (Fig. 10) et dans nos conditions opératoires, l'angle $\alpha$ formé par la verticale et le front de solidification est de $60^{\circ}$. Le passage d'une interface de solidification verticale à une interface oblique provoque une augmentation de la surface d'échange de $150 \%$.

La théorie indique que la ségrégation à l'interface a lieu normalement au front de solidification. Nous avons étudié l'influence de l'angle sur le drainage des impuretés.

Les valeurs du gradient de concentration à l'interface ont été obtenues en dérivant par rapport à $x$ et $z$ (Fig. 10) l'expression suivante :

$$
C_{\mathrm{I}}=C_{0}\left[\frac{1-k}{k}\left[1-\exp \left(-\frac{k f x}{D}\right)\right]+1\right]
$$

$C_{\mathrm{I}}:$ concentration à l'interface en $\mu \mathrm{g} / \mathrm{g}$

$C_{0}:$ concentration initiale en $\mu \mathrm{g} / \mathrm{g}$

$k$ : coefficient de partage effectif

$f$ : vitesse de déplacement en $\mathrm{cm} / \mathrm{s}$

$D$ : coefficient de diffusion en $\mathrm{cm}^{2} / \mathrm{s}$

$x$ : distance parcourue par la zone fondue $(\mathrm{cm})$

avec

$$
x_{z}=x+z \operatorname{tg} \alpha
$$

$x_{z}$ : distance parcourue par l'interface à la cote $z$

$x$ : distance parcourue par le point $\mathrm{A}$ en $\mathrm{cm}$

$\alpha$ : angle entre la verticale et le front de solidification en degré.

Le calcul ayant été effectué avec une valeur de la concentration des composantes verticale et horizontale montre que si sa valeur est indépendante de l'angle par contre l'augmentation de l'ouverture de l'angle provoque une augmentation du gradient de concentration vertical, c'est-à-dire accentue le drainage des impuretés directes vers la surface de la goutte liquide. 


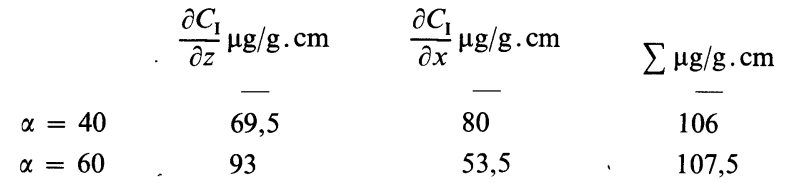

$$
\begin{gathered}
C_{0}=1 \mu \mathrm{g} / \mathrm{g} ; \quad K=10^{-4} ; \quad F=11 \times 10^{-3} \mathrm{~cm} / \mathrm{s} ; \\
D=10^{-4} \mathrm{~cm}^{2} / \mathrm{s} .
\end{gathered}
$$

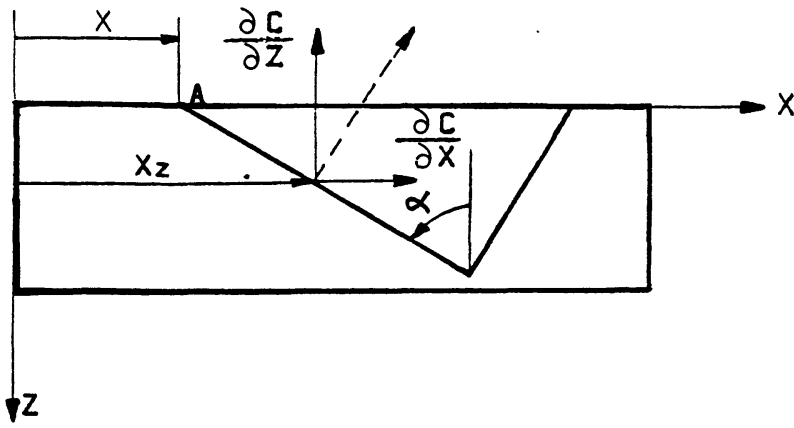

Fig. 10. - Gradient de concentration le long de l'interface.

[Concentration gradient along the interface.]

3.3 COMPARAISON ENTRE LE PROFIL DE CONCENTRATION THÉORIQUE ET EXPÉRIMENTAL LE LONG DU BARREAU. - A partir de l'ensemble des valeurs calculées précédemment nous avons comparé le profil des concentrations après deux passages en fusion de zone sous plasma et celui obtenu par le calcul théorique. Nous constatons qu'en fusion de zone sous plasma le profil expérimental est sensiblement identique au profil théorique (Figs. 11-12). Néanmoins en tête de barreau nous obtenons une amélioration par rapport à celle prévue par la théorie.

La tête du barreau présente un degré de purification supérieur à la valeur théorique. Elle traduit ainsi l'importance d'es écarts, dus aux phénomènes de drainage en surface, écarts que la théorie ne prend pas en considération. Au-delà de $3 \mathrm{~cm}$ de purification, l'accumulation des impuretés en surface modifie rapidement le phénomène de drainage.

3.4 ConClusion. - Les corrélations établies entre les grandeurs physicochimiques et les résultats expérimentaux justifient les améliorations apportées par la zone fondue au plasma. Les impuretés drainées en surface peuvent réagir avec l'hydrogène du plasma et nous avons montré que les valeurs des coefficients de partage, de l'épaisseur de la couche limite et des coefficients de diffusion sont améliorées par une température élevée et que celle-ci est importante sur la cinétique du phénomène (Tableau $\mathrm{V}$ ).

4. Résultats de la fusion de zone sous plasma. Notre travail montre que l'élaboration du silicium

Tableau V. - Influence des variables d'action sur les paramètres aux interfaces solide-liquide liquide-gaz et dans le liquide.

[Influence of work variables on the parameters at solid-liquid and liquid-gaz interfaces and in the liquid.]

+ : Favorable à la purification.

- : Défavorable à la purification.

\begin{tabular}{|c|c|}
\hline \multirow{6}{*}{$\begin{array}{c}\text { Paramètres } \\
\text { à l'interface } \\
\text { solide liquide }\end{array}$} & Surface d'échange \\
\hline & Coefficient de diffusion \\
\hline & Epaisseur de la couche de diffusion \\
\hline & Coefficient de partage effectif $k$ \\
\hline & Gradient thermique \\
\hline & $\left.\begin{array}{l}\text { Gradient de } \\
\text { concentration }\end{array}\right\} \begin{array}{l}\text { Vertical } \\
\text { Horizontal }\end{array}$ \\
\hline \multirow{7}{*}{$\begin{array}{l}\text { Paramètres } \\
\text { du liquide }\end{array}$} & Purification \\
\hline & Température du liquide \\
\hline & Coefficient de diffusion \\
\hline & Viscosité du liquide \\
\hline & Gradient thermique \\
\hline & Gradient de concentration \\
\hline & Purification \\
\hline \multirow{4}{*}{$\begin{array}{l}\text { Paramètres } \\
\text { à l'interface } \\
\text { liquide-gaz }\end{array}$} & $\begin{array}{l}\text { Elimination des impuretés par augmen- } \\
\text { tation des tensions de vapeur }\end{array}$ \\
\hline & Surface d'échange \\
\hline & $\begin{array}{l}\text { Elimination des impuretés par réaction } \\
\text { chimique }\end{array}$ \\
\hline & Purification \\
\hline
\end{tabular}

$O$ : N'intervient pas ou peu.

Purification globale
Variables d'action

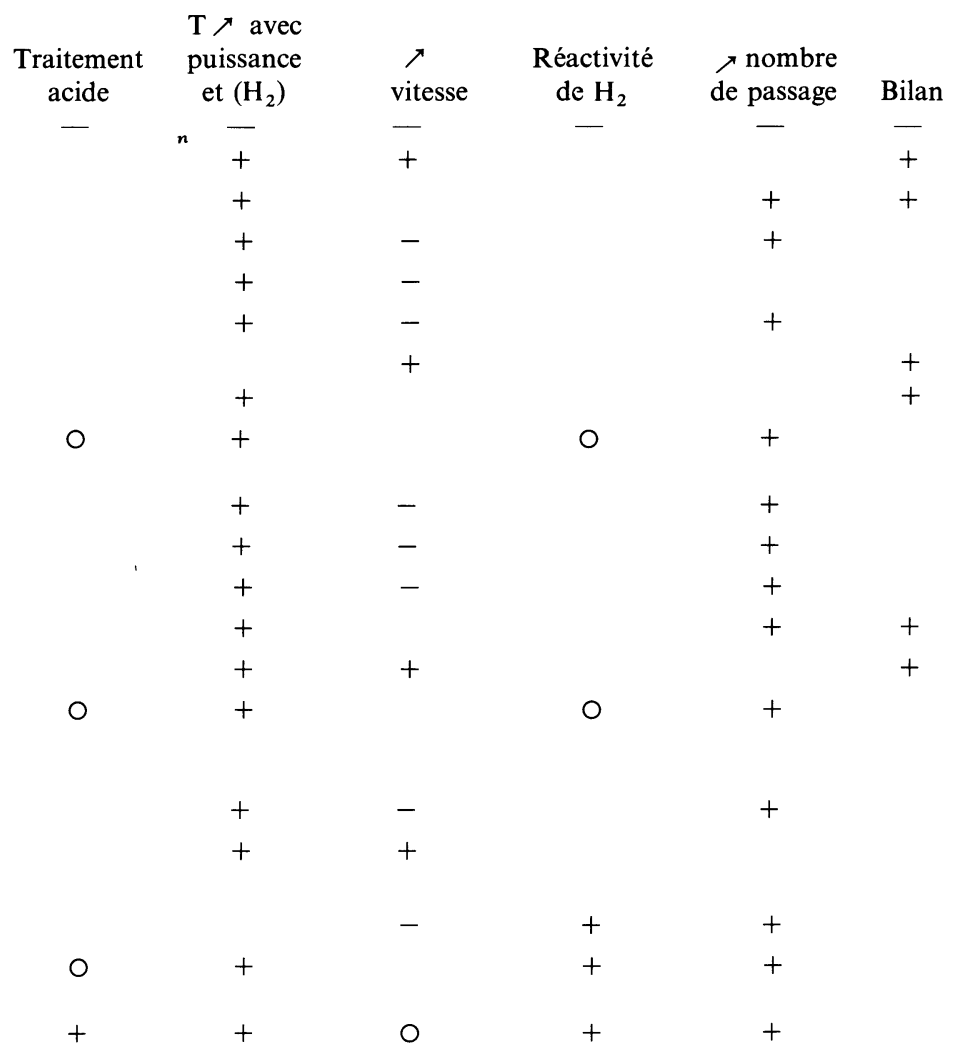


Tableau VIa. - Evolution de la teneur en impuretés en fonction des conditions de traitement. Résultats en ppm poids. a) Sans traitement acide.

[Evolution of impurities quantity as a function of treatment conditions. Results in ppm weight. a) Without acid treatment.]

\begin{tabular}{|c|c|c|c|c|c|c|c|c|c|}
\hline \multirow{4}{*}{ Eléments } & \multirow{4}{*}{$\begin{array}{c}\text { Coefficients } \\
\text { de partage } \\
\text { des impuretés } \\
\text { dans } \\
\text { le silicium }\end{array}$} & \multirow{4}{*}{$\begin{array}{c}\text { Silicium } \\
\text { métallurgique }\end{array}$} & \multicolumn{7}{|c|}{ Sans traitements acide } \\
\hline & & & \multicolumn{3}{|c|}{2 passages } & \multicolumn{2}{|c|}{4 passages } & 6 passages & 8 passage \\
\hline & & & \multicolumn{7}{|c|}{ Vitesse $\mathrm{cm} / \mathrm{h}$} \\
\hline & & & 20 & 30 & 40 & 30 & 40 & 40 & 40 \\
\hline $\mathrm{Al}$ & $2 \times 10^{-3}$ & 2800 & 200 & 1215 & 32 & 9,4 & 20 & 100 & 6 \\
\hline $\mathrm{Au}$ & $2,5 \times 10^{-3}$ & 0,001 & 0,00001 & 0,00006 & 0,0001 & 0,00002 & $<0,00002$ & 0,00003 & 0,0003 \\
\hline $\mathrm{Ba}$ & & $\begin{array}{c}10 \\
115^{*}\end{array}$ & & $<0,3$ & & $<0,2$ & $<0,4$ & $<0,4$ & $<0,27$ \\
\hline $\mathrm{Ca}$ & & 2100 & $450^{*}$ & & & & & & \\
\hline $\mathrm{Cl}$ & & $240^{*}$ & 3* & & $<0,05$ & & $<0,3$ & $<0,07$ & $<0,06$ \\
\hline Co & $8 \times 10^{-6}$ & 5 & 0,001 & 0,003 & 0,02 & 0,002 & 0,025 & 0,003 & 0,003 \\
\hline $\mathrm{Cr}$ & $10^{-5}$ & $\begin{array}{l}40 \\
75^{*}\end{array}$ & 0,04 & 0,005 & 0,16 & 0,005 & 0,08 & $<0,01$ & 0,005 \\
\hline $\mathrm{Cu}$ & $4 \times 10^{-4}$ & $40^{*}$ & 2 & & & & & & \\
\hline $\mathrm{Fe}$ & $8 \times 10^{-6}$ & 3730 & 4,5 & 0,7 & 7 & $<0,4$ & 8,4 & $<1$ & 0,6 \\
\hline $\mathrm{Mg}$ & & $\begin{array}{l}100 \\
460^{*}\end{array}$ & $23^{*}$ & $<1,8$ & $<2$ & $<1,8$ & & $<12$ & $<2$ \\
\hline Mn & $10^{-5}$ & $\begin{array}{r}84 \\
140\end{array}$ & $10^{*}$ & 0,1 & 1,8 & $<0,004$ & $<0,008$ & 0,009 & 0,002 \\
\hline Sc & & 0,99 & 0,0003 & & 0,01 & & & & \\
\hline $\mathrm{Ti}$ & $10^{-5}$ & $160^{*}$ & $25^{*}$ & & & & & & \\
\hline V & $10^{-5}$ & $85^{*}$ & $2 *$ & 0,02 & 2,1 & $<0,01$ & $<1,2$ & $<0,01$ & $<0,01$ \\
\hline $\begin{array}{l}\mathrm{Zn} \\
\mathrm{Zr}\end{array}$ & $10^{-5}$ & $\begin{array}{l}40^{*} \\
0,3\end{array}$ & $\begin{array}{r}3^{*} \\
0,02\end{array}$ & & $\begin{array}{l}<0,2 \\
0,1\end{array}$ & & & & \\
\hline
\end{tabular}

Tableau VIb. - Evolution de la teneur en impuretés en fonction des conditions de traitement. Comparaison avec le silicium polycristallin Wacker. Résultats en ppm poids. b) Avec traitement acide $\left.{ }^{*}\right)$ limite de détection.

[Evolution of impurities quantity as a function of treatment conditions. Comparison with polycristalline Wacker silicon. Results in ppm weight. $b$ ) With acid treatment $\left({ }^{*}\right)$ detection limit.]

\begin{tabular}{|c|c|c|c|c|c|c|c|c|c|}
\hline \multirow{4}{*}{ Eléments } & \multirow{4}{*}{$\begin{array}{c}\text { Silicium } \\
\text { polycristallin } \\
\text { Wacker }\end{array}$} & \multicolumn{8}{|c|}{ Avec traitement acide } \\
\hline & & \multicolumn{3}{|c|}{2 passages } & \multicolumn{3}{|c|}{4 passages } & 6 passages & $(4$ et 4$)+2$ \\
\hline & & \multicolumn{8}{|c|}{ Vitesse $\mathrm{cm} / \mathrm{h}$} \\
\hline & & 20 & 30 & 40 & 20 & 30 & 40 & $40^{\circ}$ & 40 \\
\hline $\mathrm{Al}$ & $4,5^{*}$ & 54 & 50 & & 10 & 6 & $4,8^{*}$ & $4,7 *$ & $5,4 *$ \\
\hline $\mathrm{Au}$ & 0,00005 & 0,00002 & 0,0002 & 0,002 & 0,001 & $<0,001$ & 0,00005 & 0,0001 & 0,0001 \\
\hline $\mathrm{Ba}$ & 0,15 & 0,6 & 4 & 9 & 14 & $<4$ & $<0,05$ & $<0,3$ & $<0,5$ \\
\hline $\mathrm{Ca}$ & & & & & & & & & \\
\hline $\mathrm{Cl}$ & 0,002 & & & & & $<0,02$ & $<0,1$ & $<0,2$ & $<0,2$ \\
\hline Co & 0,0007 & 0,02 & 0,06 & 0,006 & 0,1 & 0,5 & 0,01 & 0,05 & 0,001 \\
\hline $\mathrm{Cr}$ & 0,003 & 0,09 & 0,5 & 0,03 & 0,13 & 1 & 0,01 & 0,03 & $<0,01$ \\
\hline $\mathrm{Cu}$ & & & & & & & 0,04 & 0,03 & 0,03 \\
\hline $\mathrm{Fe}$ & 0,4 & 8 & 31 & 1,6 & 3 & $<6$ & $<0,8$ & $<1$ & $<0 ; 3$ \\
\hline $\mathrm{Mg}$ & & $<1,8$ & $<1,8$ & $<1,8$ & $<1,8$ & $<1,8$ & $<2$ & $<2$ & $<2$ \\
\hline $\mathrm{Mn}$ & 0,005 & 1 & 0,85 & 4,4 & 0,004 & 0,001 & 0,02 & 0,015 & 0,02 \\
\hline $\mathrm{Sc}$ & 0,00001 & & & 0,016 & 0,04 & 0,0002 & 0,00004 & 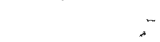 & \\
\hline $\mathrm{Ti}$ & & & & & & & & & \\
\hline V & 0,002 & 0,7 & 0,5 & 2 & 0,03 & $<0,0003$ & 0,0001 & 0,0003 & 0,008 \\
\hline $\mathrm{Zn}$ & 0,003 & & & $<1$ & $<0,2$ & $<0,03$ & $<0,001$ & & \\
\hline $\mathrm{Zr}$ & 0,24 & & & & $<1,3$ & $<0,5$ & $<0,4$ & & \\
\hline
\end{tabular}



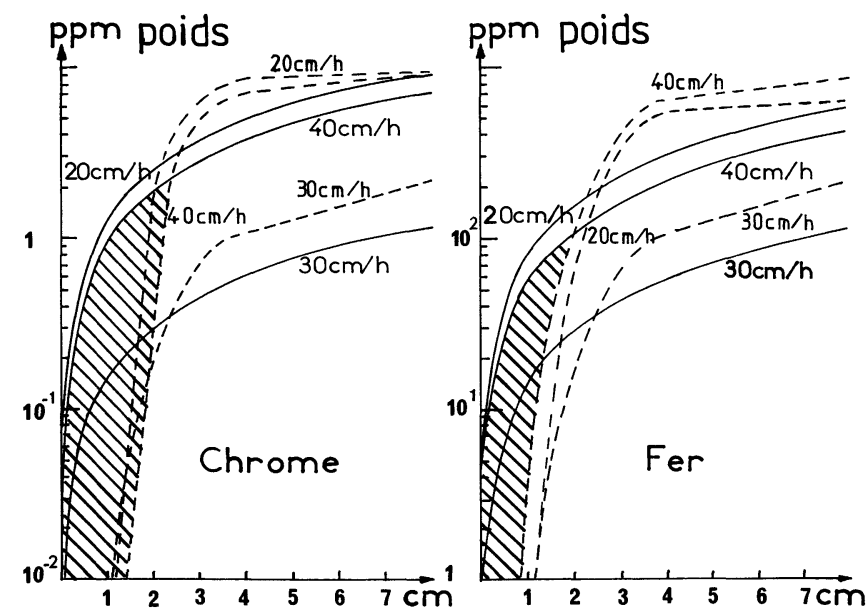

Figs. 11 et 12. - Comparaison entre les courbes expérimentales et théoriques : - - - - Courbes expérimentales. — Courbes théoriques. La surface hachurée indique la quantité d'impureté drainée à la surface du matériau après 1 passage théorique à $40 \mathrm{~cm} / \mathrm{h}$ sans décapage de la surface à l'acide. Comme nous le voyons cette quantité est considérable pour le fer et le chrome.

'[Comparison between experimental and theoric curbs :-- - - Experimental curbs. - Theoric curbs. Hashurated area indicates the impurities quantity drained to the surface of the bar after a theoritical pass at $40 \mathrm{~cm} / \mathrm{h}$ without acid cleaning. We can see that it's considerable in case of iron and chromium.]

photovoltaïque à partir du silicium métallurgique est possible par l'emploi de la fusion de zone sous plasma haute fréquence, argon-hydrogène. Les conditions expérimentales les plus favorables sont obtenues en combinant 4 passages à $40 \mathrm{~cm} / \mathrm{h}$ avec un décapage acide de la surface entre chaque traitement (Tableau VI).

L'examen cristallographique (photos 1, 2, 3, 4) montre que le silicium obtenu a des grains de 1 millimètre à quelques millimètres orientés dans le sens du déplacement de la zone fondue. Les joints de grain particulièrement fins ne révèlent aucune inclusion par contre le maclage de quelques grains est important.

Toutefois l'analyse électrique du matériau montre qu'il s'agit d'un silicium de type " $p$ » l'impureté majoritaire restante étant probablement l'aluminium ou le bore.

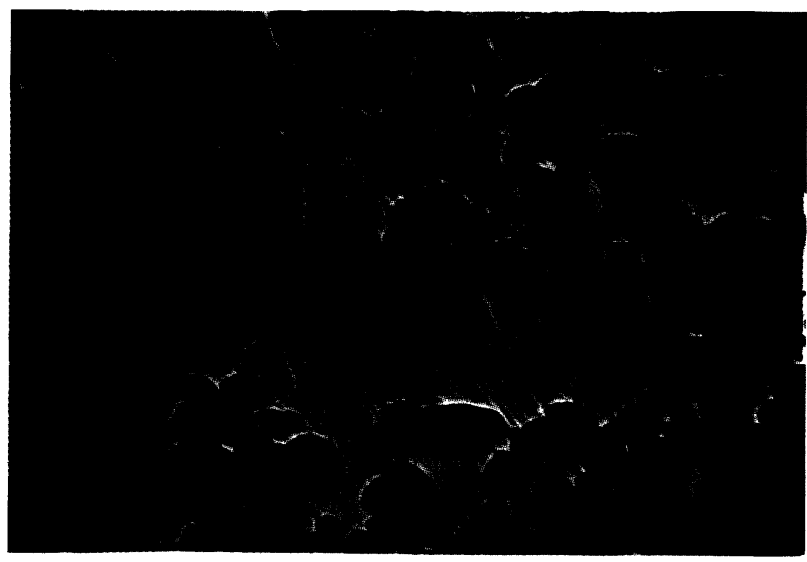

Photo 1. - Silicium métallurgique. $\mathrm{G} \times 50$.

[Metallurgical silicon. $\mathrm{G} \times 50$ ].

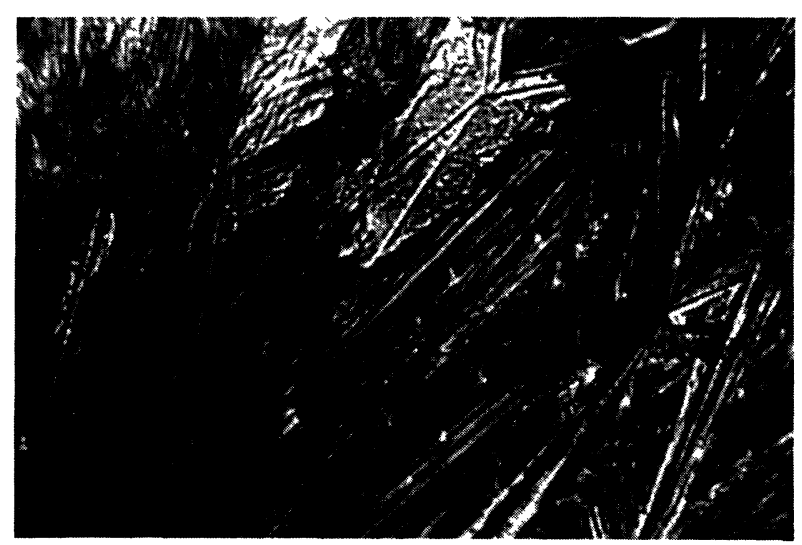

Photo 2. - Silicium polycristallin Wacker. $\mathrm{G} \times 50$.

[Polycristallin Wacker silicon. $\mathrm{G} \times 50$.]

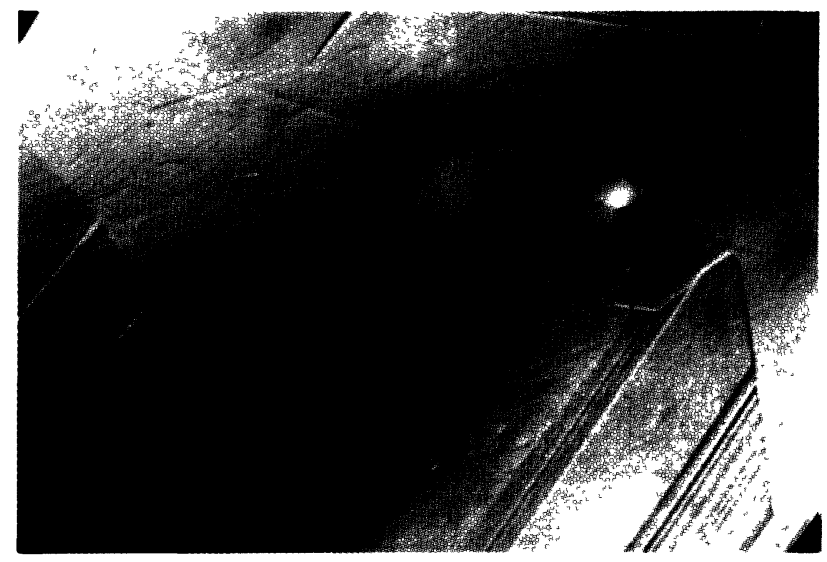

Photo 3. - Silicium polycristallin de fusion de zone sous plasma, 4 passages à $40 \mathrm{~cm} / \mathrm{h}$. Photo prise au cœur du barreau. $\mathrm{G} \times 50$.

[Polycristalline silicon obtained by plasma melting zone after 4 pass at $40 \mathrm{~cm} / \mathrm{h}$. Micrograph is taken in the middle of the bar. $\mathrm{G} \times 50$.]

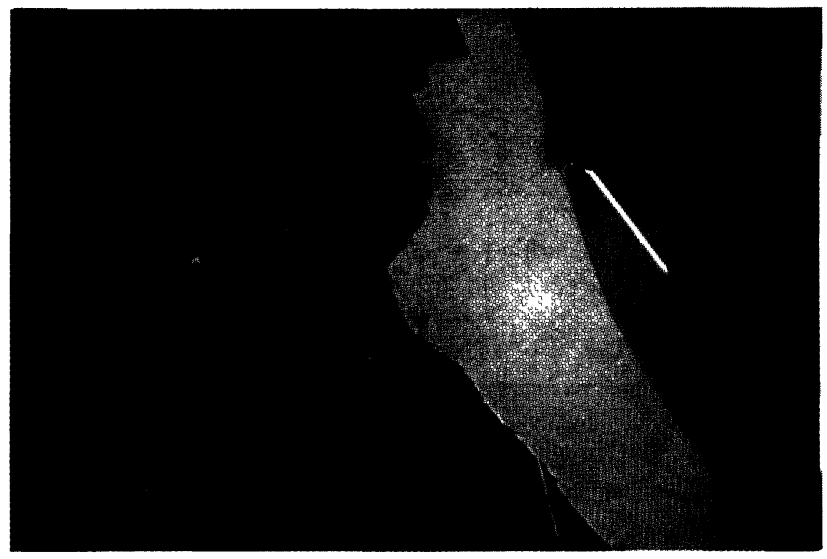

Photo 4. - Silicium polycristallin de fusion de zone sous plasma après 4 passages à $40 \mathrm{~cm} / \mathrm{h}$. Photo prise près de la surface du barreau. $\mathrm{G} \times 50$.

[Polycristalline silicon obtained by plasma melting zone after 4 pass at $40 \mathrm{~cm} / \mathrm{h}$. Micrograph is taken near the surface of the bar. $\mathrm{G} \times 50$.]

5. Conclusions. - Dans son principe cette méthode de purification conduit à l'extraction des impuretés par drainage de l'interface solide-liquide vers l'interface liquide-gaz; le transfert de matière étant dû aux 
gradients thermiques et massiques et aux courants convectifs dans la goutte liquide. Dans ces conditions la surface d'échange gaz-liquide joue un rôle déterminant qu'il s'agisse de l'élimination des impuretés à fortes tensions de vapeur ou bien de leur dissolution par un traitement acide ultérieur. Par contre la vitesse de drainage joue un rôle secondaire, elle est dictée par les vitesses de transfert de chaleur nécessaire à la fusion. Par rapport à la technique classique la vitesse de travail est multipliée par un facteur de 10 à 50 .

Le calcul des grandeurs physicochimiques respon- sables du processus de drainage permet d'interpréter en partie l'amélioration due à la technique plasma. Ainsi la modification du coefficient de partage, des coefficients de diffusion et l'augmentation de la surface d'échange (multipliée par un facteur 2,5 à l'interface liquide-solide) expliquent assez bien l'augmentation de la vitesse de transfert de matière de l'interface solide-liquide vers l'interface liquide-gaz. Ainsi le contrôle et l'optimisation d'un tel procédé apparaissent possibles dans la mesure où ces variables physicochimiques sont accessibles par l'expérience.

\section{Bibliographie}

[1] KOEPEL, S., Colloque sur les Nouvelles propriétés physiques mécaniques et chimiques du Fer de très haute pureté. Paris 1966, p. 27

[2] Le Hericy, J., C.R. Hebd. Séan. Acad. Sci. Séance du 10 octobre (1960) p. 1509.

[3] Rengstroff, G. W. P., Goodwin, H. B., Trans. AiME J. Metals 3 (1955) 467.

[4] Tadahisa, N., Tsuneaki, S., Tetsu to Hagane 55 (1969) 133.

[5] Takaki, M., Hideaki, W., Chisato, Y., Tetsu to Hagane 57 (1971) 965

[6] ReICH, R., Mem. Sci. Rev. Métal. LXII (1965) no 11.

[7] Bergheran, A., Simonsen, E. B., Trans. Metal. Soc. AIME 221 (1961) 1029.

[8] GeACH, G. A., Jones, F. O., J. the less common metals 1 (1959) 56.

[9] Purification par zone fondue, Pascal Chimie Minérale XVII (Fasc. 1) p. 544.

[10] Talbot, J., Albert, P., Chaudron, G., C.R. Hebd. Séan. Acad. Sci. Séance du 18 mars (1957) p. 1577.

[11] Wyjadlowski, T., Rochette, J., Tardy, R., Boss, J. Y., Mem. Sci. Rev. Métall. LXX (1973) 77.

[12] Fischer, J., J. Appl. Phy. U.S.A. 44 (1973) 1977.

[13] Luchar, L., Deschamps, N., Wache, A. M., Dubois, B., Dimitrov, O., Mem. Sci. Rev. Metall. LXIV (1967) no 9.

[14] Cabane, G., J. Nucl. Energy 6 (1958) 269.

[15] Pfann, W. G., Zone Melting, 2nd édition.

[16] Chaudron, G., Bull. Société Chimique de France, 26 février 1960 , p. 18 .
[17] Fedoroff, M., Monographie sur les métaux de haute pureté, Tome 3, Chap. 41, Ed. Masson, p. 523.

[18] Tiller, W. A., Jackson, K. A., Rutter, J. W., Chalmer, B. Acta Met. 1 (1953) 428.

[19] Burton, J. A., Prim, R. C., Slichter, W. P., J. Chem. Phys. 21 (1953) 1987.

[20] BRICE, J. C., The growth of crystals from the melt (NorthHolland-Amsterdam) 1965.

[21] Weinberg, F., Trans. Met. Soc. AIME 227 (1963) 231.

[22] Jillson, D. C., Sheckler, A. C., Phys. Rev. 98 (1955) 229.

[23] Cole, G. S., Winegard, W. C., J. Inst. Met. 93 (1964) 153.

[24] Wilcox, W. R., Chem. Eng. Sci. 13 (1961) 113.

[25] Dimitrov, O., Les métaux de haute pureté (P.U.F. « Le Chimiste ») 1978.

[26] Yue, A. S., J. Crystal growth 42 (1977) 542.

[27] Burris, L., Stackmann, Ch., Dillon, I. G., Trans. AIME 203 (1955) 1017.

[28] Glazov, V. M., Chizhzoskaya, S. N., Glagoleva, N. N., Liquid Crystal (Plenum Press I.N.C., New York) 1964.

[29] Grosse, A. V., J. Inorg. Nucl. Chem. 23 (1961) 333.

[30] Cavalier, G., C.R. Hebd. Séan. Acad. Sci. (1963) 1308.

[31] Murculescu, I. G., Serban, M., Rev. Roum. Chim. 19 (1974) 1417.

[32] DifFusion DATA, U.S.A., Liquid metals and alloys. Bibliographie sur la diffusion, 1972, 6, 4, 579.

[33] Chemical Engineer's Handbook, 5th Edition Perry (R. H.), Chilton (C. H.) (Mc Graw-Hill book Company). 